\title{
Le sacrifice selon Hubert et Mauss
}

Sacrifice according to Hubert and Mauss

Jean-Paul Colleyn

\section{(2) OpenEdition}

Journals

Édition électronique

URL : http://journals.openedition.org/span/274

DOI : $10.4000 /$ span. 274

ISSN : 2268-1558

\section{Éditeur}

École pratique des hautes études. Sciences humaines

\section{Édition imprimée}

Date de publication : 1 septembre 1976

Pagination : 23-42

ISSN : 0294-7080

\section{Référence électronique}

Jean-Paul Colleyn, «Le sacrifice selon Hubert et Mauss », Systèmes de pensée en Afrique noire [En ligne], 2 | 1976, mis en ligne le 04 juin 2013, consulté le 30 avril 2019. URL : http:// journals.openedition.org/span/274 ; DOI : 10.4000/span.274 
L E S A C R I F I C E

SELON HUBERTE T M A U S S
par
Jean-Paul Colleyn

\section{Introduction.}

Si le thème du sacrifice a toujours été l'objet d'une certaine fascination, l'intêrêt qu'on lui a portẻ n'est pas sans témoigner d'une certaine inconstance. La plupart des travaux systématiques qui lui ont été consacrés datent d'une époque où un certain scientisme paraissait encore avoir quelque chance de triompher en sociologie. Par la suite, on observe ce qu' on pourrait appeler un repli monographique : les contributions les plus. intéressantes portent sur des rites sacrificiels particuliers à certaines populations. Ce sujet est-il devenu tabou ? L'ethnologie s'était-elie lancée trop tôt à l'assaut de fresques ambitieuses ? L'absence d'une coupure ethnologique nette -- la notion de sacrifice subsistant dans les grandes religions occidentales -était-elle à la base d'une certaine gêne idéologique ?

Quoi qu'il en soit, le chercheur qui, aujourd'hui, se penche sur le problème trouvera comme contribution majeure et sans douce décisive sur bien des points, un texte datant de 1899 : "L'Essai sur la nature et la fonction du sacrifice", de'Henri Hubert et Marcel Mauss. Ce texte sera complèté en 1906 par "L'introduction à l'analyse de quelques phênomènes religieux" qui constitua la préface aux Mélanges d'histoire des religions (1).

(1) Ces deux textes ont été repris dans Marcel Mauss, Oeuvres complètes, I (Paris, Éditions de Minuit, 1968). 
II. Le contexte épistémologique.

L'Essai sur le sacrifice est une oeuvre précoce. A cette époque, les travaux de Hubert et Mauss font partie intégrante de 1'oeuvre collective de l'École sociologique, dont le chef incontesté était Émile Durkheim.

En 1894, celui-ci avait déjà publié les célēbres "Règles de la méthode sociologique" et il fait précéder l'"Essai sur le sacrifice" de "La dëfinition des phénomènes religieux", où il exprime de manière synthétique sa conception de l'anthropologie religieuse. Mauss, quand bien même il obéit, au cours de sa féconde carrière, à des intuitions fulgurantes et à une insatiable curiosité, n'alla jamais jusqu'à remettre en cause "l'héritage de l'oncle". Pour toute l'École d'ailleurs, c'est la transcendance du groupe sur 1'individu qui s'exprime dans la religion. Le sacré apparaît comme "l'expression symbolique du social", 1a religion n'est que "1'hypostase" de la conscience que la société se donne d'elle-même.

Quels que soient les jugements que 1'anthropologie moderne porte sur 1'axiomatique durkheimienne, il faut reconnaître qu'à 1 'époque, affirmer que les phénomènes religieux sont des phënomènes sociaux, n'était pas un lieu commun.

Si Mauss affirme avec force que "dans le sacrifice, la société est présente d'un bout à l'autre" (2), sa démarche comparative visera à dépouiller les faits sociaux de leurs traits contingents pour dégager ce qu'il appelle un type idéal. En excluant d'entrée de jeu certains matériaux et en élaborant son modèle à partir d'une matrice hébraịque et védique, Mauss limitera lui-même la portêe de son projet (3).

C'est la grande méfiance de Durkheim à l'égard des sources orales qui incita Marcel Mauss et Hubert à négliger les faits africains et amérindiens. Il faut mentionner également l'influence de Sylvain Lêvi, que Mauss appela "son deuxième oncle" et dont les travaux furent à I'origine de 1'Essai sur le sacrifice. Nos auteurs avaient également

(2) Marce1 Mauss, op. cit. I, p. 16.

(3) Il faut signaler qu'actuellement, l'interprétation des textes sur lesquels Mauss s'appuyait n'est plus admise par les indianistes. 
à l'esprit le livre de Robertson Smith, Religion of Semites, dont la deuxième édition parut en 1894 et le Golden Bough de Frazer, rédité en 1890. Marcel Mauss estima, d'autre part, que les documents sur 1 'antiquité grecque et romaine étaient insuffisants (4). Il reconnut cependant que les sources qu'il avait utilisêes présentaient un inconvénient (5); elles étaient déformées par la spéculation théologique.

Malgrë cela, Hubert et. Mauss prëtendent ẻlever leur analyse à un niveau de pertinence générale. Leur hypothèse de base est que "les faits religieux s'ordonnent en genres dont chacun est défini par une nature et une fonction, et dont les manifestations effectives ne sont que des réalisations d'un type idéal" (6).

L'inachèvement de la deuxième oeuvre de cette lignée, La prière, suggère que Mauss lui-même dut s'apercevoir que les faits de représentation mentale supportent mal la rëduction en modèles.

Pourtant Mauss ne doit pas avoir manqué totalement ses objectifs, pour qu'un ethnographe de la valeur d'Evans-Pritchard écrive dans 1a préface de l'édition anglaise de l'ouvrage, parue en 1964, qu'il s'agit "d'un essai superbe, que $l$ 'on peut considérer -- et le mot n'est pas fortuit -- (7) comme une grammaire du rite sacrificiel". Louis Dumont explique comment la systématisation de Mauss permet à l'Essai d'être encore actuel (8). Quant à Condominas, il attire 1'attencion sur la très grande valeur de l'oeuvre, comme "pense-bête", pourrait-on dire, pour 1'ethnographe de terrain (9).

III. De quelques theories du sacrifice.

Hubert et Mauss reprochent à Tylor d'avoir laissê le sacrifice

(4) Marcel Mauss, op. cit. I, p. 198.

(5) Cet inconvénient poussera Lẻvi-Strauss à exclure du domaine de 1 'analyse structurale les textes bibliques.

(6) Marcel Mauss, ibid.

(7) C'est nous qui soulignons.

(8) Cf. L'ArC, $\mathrm{n}^{\circ} 48$.

(9) Cf. Critique, $\mathrm{n}^{\circ} 301$ (juin 1972). 
sans explication après avoir imaginé une genèse des formes du sacrifice suivant le schêma : don (do ut des) $\longrightarrow$ homnage $\longrightarrow$ sacrifice de soi. Nos auteurs repoussent également l'explication gênérale proposée par Robertson Smith ; celui-ci, on le sait, faisait dériver le sacrifice de 1 a communion totémique. Lévi-Strauss mènera cette critique à son terme, soixante-trois ans plus tard (La pensée sauvage, p. 294).

Mauss objecte à Robertson Smith qu'on ne peut établir de lieu de détermination entre des phênomènes qui ne se présentent qu'exceptionnellement ensemble. Il condamne le choix arbitraire du totémisme comme point de départ d'un processus évolutif ainsi que tout essai de chronologie entre les formes du sacrifice. Mauss reproche également à Robertson Smith d'avoir exclu, par sa théorie, des rites qui constituent un des pôles du sacrifice : ceux qui ont pour effet d'expulser des êléments "pathogènes" dangereux. Pour Mauss, l'erreur majeure de Robertson Smith fut d'avoir groupé gênéalogiquement les faits d'après les rapports d'analogie qu'il croyait percevoir entre eux.

La discussion des exemples présentés par Frazer dans Le Romeau d'or permet à Marcel Mauss de proposer des limites au champ sacrificiel. I1 montre qu'il peut $y$ avoir abattage et consommation sans qu'il y ait de véritable sacrifice.

Dans l'Intichiuma australien, par exemple, bien qu'il y ait immolation et bien que le sacrê ne soit pas exempt, il manque, selon Mauss, une caractêristique essentielle du sacrifice : l'oblation, 1'attribution à des "êtres sacrés". Dans La pensée scuvage, Lévi-Strauss estimera que 1'Intichiuma n'entre pas dans la catégorie de sacrifice car il s'agit d'une opération visant à réaffirmer la convention qui est à la base d'un code, de même nature que celui mis en oeuvre pour le langage.

Accepter le critère de l'oblation implique qu'on exclue du sacrifice vrai de nombreux rites visant à renforcer la sacralité du pouvoir et notamment les cas de sacrifices humains ostentatoires, perpétrés sur des esclaves assimilés à des biens de prestige. Dans ces cas (où il est douteux que le sacrifice obéisse à un modèle cosmogonique et réalise une mise en ordre), une analyse sociologique, voire économique, du type de celle proposée par Pierre Philippe Rey (10) à propos des

(10) Cf. Colonialisme, néo-colonialisme et transition au capitalisme. 


\section{Le sacrifice selon Hubert et Mauss}

Tsangi du Congo-Brazzaville, a peut-être sa pertinence.

Chez les Kuba (Zaïre), le sacrifice humain ne semble pas avoir êtê déterminê par des considêrations religieuses. Wharton (11) rapporte que 1 'on sacrifiait des hommes par centaine pour qu'ils servent le roi dans l'au-delà.

La fonction idéologique et politique du sacrifice n'échappe pas à Hubert et Mauss. "Dans bon nombre de sociétés politico-religieuses, écrivent-ils, la hiērarchie sociale est souvent déterminée par les qualités acquises au cours des sacrifices par chaque individu. Il conviendrait aussi de considérer les cas où c'est le groupe qui est sacrifiant (...) Tous ces sacrifices de sacralisation ou de désacralisation ont sur la société (ou sur des groupes sociaux) les mêmes effets que sur 1'individu (12)."

Hubert et Mauss s'opposent aussi à Reinach (13) qui croit découvrir des totems derrière tous les dieux sacrifiés du monde gréco-romain. Ils objectent que tous les sacrifices de dieux ne sont pas totémiques et que le sacrifice des dieux n'est pas nécessairement d'origine totémique.

Signalons au passage l'intérêt d'un exemple que Mauss emprunte à Frazer : il rêvèle l'existence chez les Zuni d'une véritable chasse sacrificielle. De tels cas devraient être analysés en détail avant que soit posée une relation définitive entre la sédentarisation et le recours aux procédés sacrificiels.

Commentant Le Romeau d'or (2e éd.), Mauss conteste le bien-fondé du rapprochement opéré par Frazer entre différents sacrifices (babylonien, juif et chrétien) dont l'archétype serait le drame sacré de la végétation. Sur ce point, Mauss se borne à reconnaître que la légende chrétienne s'est formée et répandue dans un monde où on était habitué à sacrifier des dieux, à les voir mourir et ressuciter comme la nature et la végétation.

(11) The Leopard hunts alone (New York, Revel, 1927), 144 p.

(12) Marcel Mauss, op. cit. I, p. 270.

(13) Cultes, mythes et religion (1905). 
IV. Définition du système sacrificiel.

\section{A. Présupposés théoriques.}

C'est l'étude des mythes qui mène Mauss à l'êtude du sacrifice : "Dans nombre de mythes, écrit-il, où les dieux meurent pour renaître, se suicident, se combattent, sont tuês par leurs proches parents, qui se distinguent à peine d'eux-mêmes, sont alternativement victimes et sacrificateurs, l'histoire divine correspond (...) à des sacrifices rituels dont elle justifie théologiquement la célébration. Il fallait donc étudier le sacrifice pour trouver les raisons qui ont imposé ces thèmes sacrificiels à l'imagination religieuse." (op. cit. I, p. 5).

Pour Mauss, le sacrifice est au coeur du mythe et c'est par excellence un thème mythique. Cé point de vue suscite, sur le chemin d'une thêorie du sacrifice, un certain nombre de questions : Le mythe est-il toujours construit autour d'un schêma sacrificiel ? $N^{\prime} y a-t-i 1$ véritable sacrifice que quand les représentations collectives revêtent la forme du mythe ? Est-il fortuit de constater qu'auprès de certaines sociétés (Nuer, Tetela), oũ des observateurs pourtant avertis n'ont pas trouvé de représentations mythologiques, ils n'ont pas trouvé non plus de sacrifice concernant véritablement la société ?

"L'idée du sacrifice porte (ainsi) en elle le germe d'une confusion avec l'animal et qui risque même de s'étendre au-delà de l'homme, jusqu'à la divinité." (14).

C'est déjà la thèse de Mauss, qui va jusqu'à affirmer que le schéma général du sacrifice contient en puissance le sacrifice du Dieu, lequel "s'est développé après le sacrifice au Dieu et, à partir d'un certain moment, parallèlement à lui". (ibid. p. 12).

A l'époque où réfléchit' Mauss, la question des origines domine la pensée sociologique et Mauss ne peut s'empêcher de proposer une explication'bénétique" du sacrifice du Dieu. "Le point de dêpart, dit-il, est toujours, selon nous, 1'oblation (action par laquelle on offre quelque chose à la divinité), la destruction d'une chose susceptible,

(14) Lévi-Strauss, Le totémisme aujourd'hui (Paris, PUF, 1965), p.4. 
en raison des représentations qui s'y attachent, de devenir divine entre toutes les victimes sanctifiées." (ibid. p. 15).

Hubert et Mauss s'accordent avec Tylor pour estimer que le sacrifice ne peut se produire qu'assez tard dans l'évolution des religions. D'une part, il suppose la pratique du don rituel et le système des rites consécratoires, lustration, purification, etc. D'autre part, il implique et c'est là la clé de voûte de la théorie de Mauss, la séparation du sacré et du profane et la représentation mentale de "purs esprits".

"Le sacrifice est un moyen pour le profane de communiquer avec le sacrê par l'intermédiaire d'une victime." (ibid. p. 16).

Et qu'est-ce que le sacrê ? C'est le "séparé", 1'"interdit" qui "n'est pas l'effet des scrupules accumulés d'individus (..$)$; est conçu comme sacré, tout ce qui pour le groupe et ses membres qualifie la société". (íbid. p. 16).

L'Essăi sur la nature et la fonction du sacrifice a donc pour cadre la problématique durkheimienne : les choses sacrées sont celles dont la sociêté êlabore la représentation. Le fait religieux est considéré comme l'hypostase de la cohésion sociale : il réalise l'intégration symbolique de la société.

B. Définition et unité du système sacrificiel.

Sous ce titre, Hubert et Mauss jettent les bases de leur théorie formaliste du sacrifice :

"Le sacrifice implique toujours une consécration : un objet passe du domaine commun au domaine religieux." (ibid. p. 200).

Mais il y a plusieurs types de consêcration. Dans le sacrifice, la consêcration ne limite pas ses effets à l'objet sacré comme elle le fait dans 1'onction :

"La consécration rayonne au-delà de la chose consacrée $(\ldots)$ le fidèle qui a fourni la victime (...) n'est pas, à la fin de l'opération, ce qu'il était au commencement. Il a acquis un caractère religieux qu'il n'avait pas ou il s'est débarrassê d'un caractère défavorable dont il était affligê." (ibial. p. 201).

Le sacrifiant, le sujet qui subit les effets du sacrifice, précisent nos auteurs, peut être un individu ou une collectivité. 
"Le trait distinctif de la consécration dans le sacrifice, c'est que la chose consacrée sert d'intermédiaire entre le sacrifiant et la divinité." (ibid. p. 203).

Notons que dans cette optique, la distinction entre la peine religieuse et le sacrifice apparaît clairement : dans un cas la peine s'abat sur celui qui a commis le crime, dans l'autre, il y a substitution.

Il ne suffit pas, pour Hubert et Mauss, qu'il y ait oblation pour qu'il y ait sacrifice; quand il y a oblation mais sans altération de la. nature de 1 'objet consacré, il s'agit d'une offrande. Dans le cas du sacrifice, il y a destruction ou altération de l'objet du sacrifice. La définition de Mauss n'admet cependant pas que 1 'on restreigne, come semble parfois le faire l'usage, le sens du mot sacrifice en le rêservant à la désignation des seuls sacrifices sanglants. Et en ce qui concerne ceux-ci, nos auteurs estiment que le sacrifice humain doit être étudié comme tous les autres.

L'analyse qui précède amène les auteurs de l'"Essai sur le sacrifice" à proposer, dans le style un peu "juridique" qui est le leur, la définition suivante :

"Le sacrifice est un acte religieux qui, par la consêcration d'une victime, modifie l'état de la personne morale qui l'accomplit ou de certains objets auxquels elle s'intéresse." (ibid. p. 205).

Cette définition suppose l'unité générique des sacrifices sans en nier la diversité. Pour Hubert et Mauss, "il y a continuitẻ entre les formes du sacrifice. Elles sont à la fois trop diverses et trop semblables pour qu'il soit possible de les diviser en groupes trop caractérisés" (ibid. p. 211). C'est la raison pour laquelle les auteurs de 1"'Essai" ne prennent à leur compte aucune classification couramment proposée (et notamment celle de l'École allemande qui distingue les sacrifices expiatoires, les sacrifices d'action de grâce, les sacrifices de demande, etc.). Est même exclue la division en deux idées irréductibles d'expiation et de communion, de communication d'un caractère sacré d'une part et d'expulsion d'un caractère contraire de 1 'autre. Nos auteurs n'acceptent donc pas la distinction reprise en des termes plus neutres par Luc de Heusch en rites de disjonction et rites de conjonction :

"Peut-être chercherons-nous en vain des exemples de sacrifices 
expiatoires où ne se glisse aucun élément communiel ou de sacrifices communiels qui ne ressemblent par aucun côtê à des sacrifices expiatoires." (ibid. p. 211 ).

c. Le "schème" sacrificiel.

Sous ce titre, à la consonance un peu kantienne, Hubert et Mauss tentent, au-delà des formes particulières, de démonter le mécanisme du sacrifice. Ils ne prétendent cependant pas à un degré de pertinence absolu.

"Nous ne pouvons évidemment songer à dessiner un schème abstrait qui soit assez complet pour convenir à tous les cas connus (...) Tout ce qu'il est possible de faire, c'est d'étudier des formes de sacrifices assez complexes pour que tous les moments importants du drame y soient rêunis (...) Le sacrifice qui nous parâ̂t le mieux répondre à cette condition est le sacrifice animal hindou védique." (ibid. p. 212).

(a) Les rites d'entrée.

Pour établir une communication avec le monde sacré, il faut augmenter le caractère religieux, sacré des agents du sacrifice. Il faut également que cette approche du sacré soit entourée de précautions. Ainsi s'expliquent les éléments rituels suivants :

-- la purification du sacrifiant : abstinence, toilette rituelle, vêtements spéciaux, interdits, parfois mort symbolique, etc.

-- le sacrificateur est parfois un prêtre qui, par définition, est au seuil du monde sacrê et du monde profane.

-- les règles qui concernent le lieu et les instruments du sacrifice : tous les moments du jour et de $I^{\prime}$ année ne sont pas propices. Le lieu est un sanctuaire, un enclos ou un endroit préalablement consacré, qui abrite souvent des autels. La victime doit avoir certaines qualités pour être consacrée ; on prend certaines précautions pour qu'elle ne devienne pas dangereuse car elle est un "foyer d'énergie".

(b) L'immolation.

Tout en s'avançant dans le monde des dieux, la victime doit rester en relation avec les hommes : les rites ont pour fonction d'assurer la communication. Après Hubert et Mauss, Lévi-Strauss insistera sur la consubstantialitê du sacrifiant, de la victime et de la divinité : 
"Tout sacrifice implique une solidarité de nature entre l'officiant, le dieu et la chose sacrifiêe." (Lévi-Strauss, Le Totémisme aujourd'hui, p. 5).

C'est pourquoi 1'opération suprême est un crime, une sorte de sacrilège ; certains rituels exigent des libations, des expiations. On s'excuse de l'acte à accomplir. Mais par ce meurtre, on dégage une force redoutable qu'il faut dompter, diriger. Le corps de l'animal est chargé d'une force sacrée. Matière sacrée, il va servir à développer les effets utiles du sacrifice tout en étant attribué tout entier au monde sacré, tout entier au monde profane ou partagé entre les deux. Les rites sacrificiels s'accompagnant de destruction complète auraient souvent pour fonction d'éliminer, d'expulser, bien que l'idée d'attribution n'en soit pas absente. Quand les restes de la victime ne sont pas tout entiers attribués aux dieux, ils communiquent aux sacrifiants ou aux objets du sacrifice des vertus religieuses dont les avait déchargës la consëcration sacrificielle. La victime passe aux sacrifiants les qualités nouvelles qu'elle a acquise par la sacrification.

Les rites opérant le contact entre certaines parties du corps de 1 'animal et l'autel d'une part et entre certaines parties et le sacrifiant de l'autre réalisent la communication. Citons parmi ces rites les aspersions de sang, le dêversement de sang ou d'aliments dans l'eau pour les divinités aquatiques, le port de la peau, des entrailles de la victime sacrificielle, l'incinération de la victime (comunication par la fumée), la "part des dieux" dans les banquets sacrificiels, etc. Hubert et Mauss notent qu'un sacrifice sanglant n'a pas nécessairement pour principe 1 'usage du sang : dans certains rituels indiens, on laisse le sang s'échapper (pour le démon) et la partie consacrée au Dieu est un organe interne (le coeur) qui est brûlé.

Le schêma est donc le suivant : on consacre la victime puis on laisse s'ëchapper, les unes vers "les êtres du monde sacré, les autres vers les êtres du monde profane", les énergies que cette consécration a suscitées. Parfois l'immolation suffit à "dēcharger" ia victime mais souvent, il faut de nouveaux rites pour éliminer vers le sac̀ré un excès de force redoutable.

"(La victime) est chargée d'une telle sainteté que le profane, malgré les consécrations préalables qui l'ont, dans une certaine mesure, élevé au-dessus de sa nature ordinaire et normale, ne peut y toucher 
sans danger, il faut donc abaisser de quelques degrés cette religiosité qui est. en elle et qui la rend inutilisable pour de simples mortels." (Mauss, op. cit. I, p. 250).

(c) Les rites de sortie.

L'immolation n'est donc pas la fin du sacrifice. Il faut, pour retrouver la vie normale, dêsacraliser les éléments du rite. Entrent dans ce cadre les opêrations suivantes :

-- destruction des instruments rituels,

-- 1ibations pour se purifier des fautes rituelles,

-- lavage (eau lustrale),

-- changement de vêtements, etc.

(d) La variabilitê des formes du sacrifice.

"Suivant la fin poursuivie, suivant la fonction qu'il doit remplir, les parties qui le composent peuvent se disposer suivant des proportions différentes et dans un ordre différent; les unes peuvent prendre plus d'importance au détriment des autres, certaines même peuvent faire totalement défaut. De là naît la diversité des sacrifices." (ibid. p. 256).

Nous aurons donc à envisager plusieurs cas. Si avant le sacrifice le sacrifiant n'est investi d'aucun caractère sacré (dans le cas d'ordination, d'initiation par exemple), les rites d'entrée sont très développés. Ces sacrifices doivent modifier la personnalité du sacrifiant. "Il faut, écrivent Hubert et Mauss, que le sacrifiant garde quelque chose de ce qu'il a acquis au cours du sacrifice. Les pratiques de sortie sont donc réduites à leur plus simple expression." (ibid. p. 256).

Souvent, les rites d'initiation sont, pour reprendre un concept proposé par Luc de Heusch, des rites d'adorcisme : ils ont pour objet "d'introduire une âme dans un corps" (p. 257).

D'une manière générale, Hubert et Mauss appellent "sacrifices de sacralisation", ceux dont le but est soit de "crêer de toutes pièces un caractère sacrē chez le sacrifiant", soit "simplement d'augmenter un caractère préexistant" (p. 258). Dans ce cas c'est après l'immolation que se déroule le rite le plus important : sa modalité extrême est la communion alimentaire. Nous retrouvons donc ici les procédés qui visent à assurer une conjonction. Parfois au contraire 1 'accent est mis sur la disjonction. Il s'agit du sacrifice de désacralisation, de 
purification ou d'expiation. La transmission doit ici aller du sacrifiant à la victime et non plus de la victime au sacrifiant. Les rites d'entrée sont alors réduits car la souillure est une sorte de consécration. "Le pécheur, comme le criminel (on pourrait ajouter : et le possédé), est un être sacré." (íbid. p. 258).

Le sacrifiant, déjà investi de sacralité, n'a rien à acquérir ; il s'agit de se débarrasser de certaines forces sur la victime. Aussi "la religiosité s'abaisse-t-elle progressivement depuis le commencement de la cérémonie" (p. 261). Il ne s'agit pas nécessairement d'éliminer des forces maléfiques : lorsqu'un prêtre veut se délier d'un voeu ou quitter un état de grande sainteté qui pourrait être dangereux, un sacrifice est souvent nécessaire.

L'offrande des prémices apparaît également comme un rite sacrificiel de désacralisation. "Chaque espèce de fruits, cêréales et autres est toute entière sacrée, interdite, tant qu'un rite, souvent sacrificiel, n'en a pas fait disparâtre l'interdit qui la garde." (ibid. p. 263).

Avec ces distinctions entre différentes modalités, nous sommes loin cependant de la classification en catégories irrêductibles : "Il ne serait pas tout à fait exact de se représenter 1'expiation comme une élimination pure et simple où la victime ne jouerait que le rôle d'un intermédiaire passif ou d'un rêceptacle. Elle se charge d'une consêcration qui n'est pas toujours différente de celle qu'elle prend dans les sacrifices de sacralisation. Aussi bien verrons-nous des rites de sacralisation et des rites expiatoires réunis dans un même sacrifice. La force que contient la victime est de nature complexe (...) C'est qu'en effet, comme l'a bien montré Robertson Smith, le pur et l'impur ne sont pas des contraintes qui s'excluent; ce sont deux aspects de la vie religieuse. (...) Les forces religieuses peuvent s'exercer pour le bien comme pour le mal $(\ldots)$, on s'explique ainsi comment le même mécanisme sacrificiel peut satisfaire à des besoins religieux dont la différence est extrême. Il porte la même ambiguité que les forces religieuses elles-mêmes (...) La victime représente aussi bien la mort que la vie, la maladie que la santé. (...) Elle est le moyen de concentration du religieux, elle l'exprime, elle l'incarne, elle le porte. C'est en agissant sur elle qu'on agit sur lui $(\ldots)$ soit qu'on l'attire, (...) soit qu'on l'expulse. On s'explique de la même manière que $(\ldots)$ ces deux formes de religiosité puissent se transformer l'une dans l'autre." 
(ibid. pp. 265-266).

Une autre variable détermine la forme du sacrifice. Quand 1'opëration concerne la personne même du sacrifiant, celui-ci est "1'origine et la fin du rite". Il arrive alors que la communion détermine une modification ou une régênêrescence de la personnalitê du sacrifiant.

Quand l'effet du sacrifice porte sur un objet autre que le sacrifiant, les rites d'entrëe et de sortie (qui ont particulièrement en vue le sacrifiant) deviennent rudimentaires et la sacrification prend dès lors plus de place.

Dans les sacrifices de fondation, l'importance de la victime varie selon qu'il s'agit d'un sanctuaire, d'une maison ou d'un village. Suivant que l'édifice est déjà construit ou à construire, le sacrifice aura pour objet de "créer l'esprit" ou de propitier le gênie du sol.

Les sacrifices agraires fournissent à Hubert et Mauss l'occasion de démontrer comment différents mécanismes peuvent jouer dans un sacrifice unique. Portant en effet essentiellement sur des objets, ils $n$ 'en n'ont pas moins des effets sur le sacrifiant.

"Parfois, on se représente (ce principe religieux qui sommeille en hiver) comme un esprit qui monte la garde autour des terres et des fruits ; il les possède et c'est cette possession qui constitue leur sainteté. Il faut donc l'êliminer pour que la moisson soit possible. Mais en même temps, comme il est la vie même du champ, il faut, après l'avoir expulsé, le recréer et le fixer dans la terre dont il fait la fertilité (...). Les sacrifices agraires ont donc, pour la plupart, des effets multiples." (ibiä. p. 274).

I1 Eaut remarquer que les sacrifices agraires présentent fréquemment une série ininterrompue de concentrations et de diffusions.

"Aussitôt la victime devenue esprit, génie, on la partage, on la disperse pour semer la vie avec elle. Pour que cette vie ne se perde pas (...) il faut la rassembler périodiquement." (ibid. p. 282).

\section{Le sacrifice du Dieu.}

On vient de voir comment Hubert et Mauss ont étudiê un assemblage de variables fonctionnellement reliêes, un peu à la manière dont on étudierait, au-delà des formes particulières, les attributs généraux. 
d'un mouvement mécanique.

En abordant le sacrifice du Dieu, qu'ils considerent comme une des formes les plus achevées de l'évolution historique du système sacrificiel, nos auteurs prennent, sur le plan théorique, davantage de risques :

"C'est dans le sacrifice d'une personne divine que la notion de sacrifice arrive à sa plus haute expression." (ibid. p. 283) (Nietzsche aurait ajouté "et à son degré suprême de séduction").

L'analyse proposée vise surtout à éclairer les rapports entre la mythologie et le sacrifice.

"Pour qu'un Dieu puisse ainsi descendre au rôle de victime, il faut qu'il y ait quelque affinitê entre sa nature et celle des victimes. Pour qu'il vienne se soumettre à la destruction sacrificielle, il faut qu'il ait son origine dans le sacrifice lui-même. Cette condition paraît, à certains égards, remplie par tous les sacrifices, car la victime a toujours quelque chose de divin que dégage le sacrifice. Mais une victime divine n'est pas une victime dieu." (ibid. p. 284).

Mannhard et Frazer avaient mis en relation, dans une perspective évolutionniste, sacrifices agraires et sacrifice de la divinité. Sans soutenir que tout sacrifice du Dieu soit d'origine agraire, Hubert et Mauss tentent de démontrer que les sacrifices agraires ont pu fournir un point de départ à l'apparition du sacrifice du Dieu. Ils croient pouvoir établir que peu à peu "l'âme de la vie des champs" se distingue de ceux-ci, devient autonome, s'individualise.

"(L'association entre un être) et une vertu surnaturelle est le fruit de la périodicité des sacrifices agraires. La répétition de ces cérémonies, dans lesquelles (...) une même victime reparaissait à intervalles réguliers, a créé une sorte de personnalitê continue. (...) Le sacrifice a fourni les éléments de la symbolique divine.(...) L'imagination des créateurs de mythes a (...) donné une histoire, et partant, une vie continue à la personnalité intermittente, terne et passive qui naissait de la périodicité des sacrifices." (ibíd. p. 288).

Ce n'est pas tout : "Les mythes conservent la trace de leur origine : un sacrifice plus ou moins dénaturé forme l'épisode central et comme le noyau légendaire des dieux qui sont sortis du sacrifice." (ibia. p. 288).

Les combats divins ne sont pas autre chose qu'une des formes 
Le sacrifice selon Hubert et Mauss

mythologiques du sacrifice du Dieu. Les protagonistes de ces combats sont d'ailleurs souvent le produit du dêdoublement d'un même génie : "ils sont présentés comme des combats météorologiques entre les dieux du ciel et de l'enfer. Mais il est extrêmement difficile de distinguer avec netteté le caractère de chacun des combattants. Ce sont des êtres de même nature dont la différenciation, accidentelle et instable, appartient à 1'innagination religieuse." (ibid. p. 293).

Hubert et Mauss établissent un schéma "généalogique" sacrifice $\longrightarrow$ mythologie $\longrightarrow$ sacrifice du Dieu, ce qui leur permet de dire : "Le mythe une fois constitué réagit sur le rite d'où il est sorti et s'y rêalise. (...) Il s'ensuit que le sacrifice apparaît comme une répétition et une commémoration du sacrifice originel du Dieu." (ibia. p. 296).

Quelles que soient les réserves formulées à propos d'une succession historique de formes sacrificielles, l'analyse du sacrifice du Dieu proposée par Hubert et Mauss ne peut manquer, si on la confronte par exemple aux mythologies du monde mandé, d'éveiller le vif intérêt des africanistes.

\section{Conclusion.}

Au terme de cet itinéraire, il nous faut maintenant reposer les questions fondamentales : le sacrifice est-il une institution objective? Ne doit-il pas son unité à une catëgorisation abusive, à une réification que l'analyse rigoureuse des faits ne permet pas de justifier ? Ou encore ne doit-on pas ramener le sacrifice à une multiplicité de formes irréductibles ? Ne doit-on pas alors condamner tout effort de définition absolue et se contenter de la description de ces formes ?

Voyons quels types de réponse la méthode inductive de Hubert et Mauss, qui vise à atteindre, au-delà de la complexité empirique, quelques principes simples, permet de fournir.

Quelle que soit la diversité des formes, le sacrifice leur apparaît toujours comme "un procédé qui consiste à établir une communication entre le monde sacré et le monde profane par l'intermédiaire d'une victime, c'est-à-dire d'une chose détruite au cours de la cêrêmonie." (ibid. p. 302). 
Le profane entre en relation avec le sacré. Mais la communication est médiatisée car le contact des forces sacrées est redoutable. Si le sacrifiant s'engageait tout entier dans le rite, il y trouverait la mort, non la vie; la victime le remplace. "Si le sacrifiant donne quelque chose de soi, il ne se donne pas ; (..) s'il donne, c'est en partie pour recevoir. (...) Au fond il n'y a peut-être pas de sacrifice qui n'ait quelque chose de contractuel." (ibid. p. 305).

Dans tout cela, il y a quelque chose qui dérange. On a presque envie de dire, avec le philosophe, "qu'ils sentent un peu mauvais ces ateliers où 1 'on fabrique le sacré". Car tout se passe de la façon dont Hubert et Mauss mènent leur êtude, comme si les théories du sacrifice étaient vraies. Or, comme le remarquent Lëvi-strauss (La pensée sauvage) et Girard (La violence, et le sacré), dans la communication, il y a un terme qui est imaginaire : la divinité. Ce n'est pas étudier un donné que de considérer comme le dernier mot à son propos, le discours qu'il se donne lui-même. Étudier les formes du sacrifice uniquement, c'est donner corps au mythe, c'est accepter une dissimulation initiale. Il est vrai que Mauss affirme que : "L'explication sociologique est terminée quand on a vu ce que les gens croient et pensent et qui sont les gens qui croient et pensent cela".(L. Dumont, L'Arc, 48,p.65).

Il serait faux cependant de juger rapidement et de dire qu'en défendant la théorie de la médiation, Hubert et Mauss se comportent comme s'ils croyaient aux représentations mentales qu'ils étudient. Pour eux, en un certain sens, la divinitê existe, mais conformément à la thèse durkheimienne, comme "force collective hypostasiée".

"On pourrait croire que le système (sacrificiel) tout entier n'est qu'un jeu d'images. Les pouvoirs auxquels s'adresse le fidèle qui sacrifie ses biens les plus précieux, semblent n'être rien de positif (...). Qui ne croit pas ne voit dans ces rites que de vaines et coûteuses illusions et s'étonne que toute l'humanité se soit acharnée à dissiper ses forces pour des dieux fantomatiques." (Mauss, ibid. p. 305).

Mais les représentations mentales, rëpondent immédiatement Hubert et Mauss, existent objectivement comme des faits sociaux. Abstraction faite de ses formes, le sacrifice rappelle aux consciences particulières, les forces collectives et entretient leur existence idéale.

"Ces expiations, ces purifications, ces communions, ces sacralisations, ces créations de gênies donnent et renouvellent périodiquement 
à la collectivité, représentée par ses dieux, ce caractère bon, fort, grave, terrible qui est un des traits essentiels de toute personnalité sociale." (ibid. p. 306).

Ainsi, en tant que transfiguration de la société, le sacré appartient au domaine des idées. Mais il n'est pas entièrement extérieur aux individus car "derrière ces idées, il y a du respect, de l'amour, de la répulsion, de la crainte, des sentiments divers et forts, évocateurs, de nature à se traduire en gestes et en pensée." (ibid. p. 17).

Quoi qu'il en soit, la question primordiale demeure : pourquoi le sacrifice? Pourquoi ce parti-pris de malheur et de violence nécessaire dans les traditions humaines ? Pour y répondre, il faudrait d'abord que soit élucidée la notion de sacré.

L. de Heusch : I1 y a au fond deux théories complëmentaires chez Hubert et Mauss ; $j$ 'aimerais les distinguer. La première nous présente le sacrifice comme un rite de passage subi par le sacrifiant, individu ou collectivité ; la seconde expose deux mécanismes formels opposés, selon que 1'on envisage le sacrifice sous l'angle de la "sacralisation" ou de la "désacralisation". Ces deux termes sont équivoques. Il n'en demeure pas moins que les auteurs décrivent deux fonctions rituelles polaires, dont les religions africaines jouent effectivement. Je ne crois pas m'ëloigner fondamentalement de la pensẻe d'Hubert et Mauss en proposant de qualifier respectivement ces fonctions de "conjonctrice" et "disjonctrice", en me plaçant à un niveau à la fois plus neutre et plus général.

En revanche, je ne suivrai pas Hubert et Mauss lorsqu'ils s'ingénient à mettre en corrélation leurs deux théories : en effet, la première, l'interprétation du sacrifice comme rite de passage, me paraît d'un faible secours analytique en Afrique.

A. de Surgy : Qu'entendent les auteurs par cette partition sacré/ profane, qui paraît un peu gratuite ? Que représentent ces forces hypostasiées?

J. $-P$. Colzeyn : Mauss semble beaucoup tenir à cette idée, car c'est elle qui lui fournit son point de départ, que ce soit pour le sacrifice, pour la prière ou pour 1 'interdit; mais il ne $s^{\prime}$ attarde pas sur $1^{\prime}$ explication de ces forces collectives.

A. Adzer : Une chose parait flove, c'est la coupure si nette entre le sacrifice avec victime, donc avec altération de la chose offerte et 1 'offrande proprement dite. Si $1^{\prime}$ on se place dans la perspective de la théorie de la communication entre deux mondes, comment peut-on négliger le continuum entre ce qui est offrande et victime sacrificielle ? Pourquoi 1'altération ? Est-elle le propre du sacrifice par opposition au don, à l'offrande ? 
L. de Heusch : Dans 1'optique de Mauss, le sacrifice serait une offrande d'une nature spéciale à cause de l'opération de consécration et de destruction.

M. Cortry : Pour qu'il y ait altération, il faut qu'il y ait participation de la victime et métamorphose de l'objet qui rayonne sur le sacrifiant.

L. de Heusch : Les Thonga, tribu bantoue d'Afrique australe, mettent sur le même plan linguistique offrande et sacrifice. La distinction $n$ 'est pas faite. Le principe de substitution joue ici pleinement : par exemple, un morcèau de peau peut être offert à la place d'un boeuf. La valeur de 1 'offrande importe peu.

La perspective sociologique de Mauss prend du poids dans le contexte thonga : le sacrifice sanglant, extrêmement élaboré, intervient lorsque des relations de groupes sont en jeu. Dans les rites de fin de deuil, et les rites de mariage, le sacrifice permet d'exprimer et d'apaiser tout à la fois. La problématique de Max Gluckmann pourrait compléter ici de manière heureuse celle de Hubert et Mauss. Mais la distinction entre "offrande" et "sacrifice" n'apparaît que dans la pratique rituelle, non dans la langue.

D. Jonckers : Chez les Minyanka, il n'y a qu'un seul terme, qui veut dire "donner", pour désigner 1 'offrande ou le sacrifice sanglant. Par contre, il y a des termes différents pour distinguer sacrifices collectifs ou individuels, ou les motivations particulières (demande, remerciement, etc.)

L. de Heusch : Mauss n'explique pas très clairement comment les sacrifices personnels expriment la communauté toute entière. Le sacrifice du boeuf est, chez les Nuer, un rite de "désacralisation" qui ne concerne que l'individu.

A. Adzer : Je crois que, chez les Nuer, il y a, dans le troupeau, des vaches qui sont réservées pour servir aux sacrifices. Cela suppose un consensus du propriêtaire et du lignage. Le sacrifice, par 1à même, intéresse tout un groupe.

J.-P. Colzeyn : Pour Mauss, le sacrifice est toujours social, qu'il intêresse l'individu ou la société. On ne peut pas sacrifier de manière asociale, même lors des sacrifices à caractère magique. Le sacrifice est comme la parole. Dans chacun, il y a une convention collective. Individuel s'oppose à collectif et non à social.

L. de Heusch : Mauss a eu le mérite, en tout cas, d'interpréter les offrandes de prémices comme des sacrifices de dêsacralisation collective. Cette thêorie mériterait d'être discutée.

J.-P. Colleyn : Dans certains cas la mythologie explique clairement que la nouvelle récolte est sacrée.

J.-E. Vincent : On peut aussi interpréter cela comme un sacrifice de remerciement aux divinités responsables de la récolte.

A. Adler : Apparaît ici l'idée que la consommation de la récolte est liée à l'extraction d'une force sacrée.

G. Le Moal : Le sacré est une force dangereuse. Chez les Bobo, cultiver c'est aller contre 1'ordre naturel du monde. La brousse est un lieu qui ne devrait pas être cultivé. Cultiver, c'est violer la terre. 
Le sacrifice selon Hubert et Mauss

J.-P. Colteyn : Chez les Bambara, au contraire, cultiver c'est purifier la terre.

G. Le Moat : Le raisonnement est inverse. Pour rendre consommable la récolte, il faut lui enlever sa souillure. Chez les Bambara, pour pouvoir être consommée, la récolte doit être désacralisée.

M. Cartry : Mme Dieterlen parle des rites de prémices chez les Dogon dans une perspective de désacralisation. Le mil n'est pas conscmmable tant qu'on n'a pas extrait son âme. Par contre, pour les semailles, il faudra réintégrer l'âme dans la graine.

G. Le Moal : Un autre raisonnement consiste à dire : couper le mil, c'est le tuer, c'est libêrer son âme. Il faut donc faire des opérations sur son nyama pour recanaliser la force vitale et redresser les choses.

Y. Cissé : Je crois que les croyances des Bambara et des Minyanka rejoignent 1 'idée de Hubert et Mauss relative au sacrifice du dieu. La victime sacrificielle doit porter en elle une parcelle divine. Seule cette parcelle compte et est sacrifiêe. Ce qui explique l'importance du choix des victimes (taches, anomalies, etc.).

A. Adler : Il y a donc fusion entre la victime et le destinataire.

Y. Cissé : L'idée avancée n'est-elle pas inscrite, chez les Gourmantché, dans le choix des poulets sacrificiels?

M. Cartry : Chez les Gourmantché, le choix du poulet correspond à un langage. I1 renvoie à des types bien dëfinis de demande. La divinité ne peut authentifier un sacrifice que s'il revêt la bonne forme. C'est pour cela qu'il faut fixer la demande par une écriture.

L. de Heusch : Est-ce que le sacrifice est toujours un rite de passage? J'en doute.

J.-F. Vincent : On peut peut-être aussi parler de passage quand il y a retour à un état initial. Le sacrifice qui a pour but la guérison d'un malade le fait simplement revenir à un état antérieur.

A. Adler: Je ne pense pas. Le malade guéri a toujours quelque chose de plus qui le marque et le fait appartenir à un groupe.

L. de Heusch : Pas toujours. Chez les Thonga, par exemple, les possédés exorcisês appartiennent à la confrêrie des devins et des voyants et sont capables de guérir un certain nombre d'affections mentales. Par contre, chez les Nuer, le malade guéri grâce au sacrifice revient à son état initial. Il y a simplement remise en ordre et non passage.

A. de Surgy : Ce qui semble faire défaut dans la thêorie d'Hubert et Mauss, c'est le côté théologique. Il ne distingue pas les différentes variétés de dieux. Or, s'il est question de signifier quelque chose en relation avec la divinité, c'est évidemment très important.

J. $-F$. Vincent : Il m'a paru paradoxal, en effet, qu'il ne soit jamais question des forces auxquelles s'adresse le sacrifice.

A. Adler : On a l'impression que, dans cette thêorie, on ne parle du sacrifice que comme quelque chose qui donne directement un acquis au sacrifiant ou à la collectivité. Ne pourrait-on pas dire aussi que le sacrifice accrô̂t le pouvoir de la divinitê, et par conséquent intéresse cette dernière plus que la collectivité elle-même ? 
L. de Heusch : Mauss dit que le sacrifice rejaillit tantôt sur le sacrifiant tantôt sur la divinitê elle-même. Mais il n'explicite guère, en effet, ce second aspect.

M. Cartry : I1 y a cette notion de "fétiches", si difficile à manier. Il suffit parfois d'un sacrifice sur un objet indéterminé pour faire apparaître cêt objet comme "fétiche", sans être obligé de postuler au départ un vague esprit qui choisirait cet objet comme support. Il y a aussi ces fameux boli ou buli. On ne peut pas dire au départ qu'il y a des entités flottantes, sans support, qu'on appellerait esprits, auxquelles on affecte tel ou tel support, tel ou tel objet. Il $y$ a un rite; c'est tout ce qu'on sait. L'apparition d'une nouvelle catégorie dans le système des buzi gourmantché est intrinsèquement liêe à l'objet sur lequel on a sacrifié.

A. Adler : Est-ce n'importe quel objet ?

M. Cartry : Non. Cela dépend de tout un système.

A. Adler : quand un lieu est sacré, on postule qu'il y a une entité préalable; quand l'autel est fabriqué, on crée une nouvelle entite..

G. Le Moal : En fait, on rend favorable un objet ou un lieu à la fixation d'un esprit qui est déjà ailleurs.

L. de Heusch : Peut-on dire ici que le sacrifice est créateur ?

M. Cartry : Non, pas vraiment. Par ailleurs, 1 'objet sur lequel on sacrifie ne peut pas être apprêhendé comme le support ou le réceptacle d'un esprit.

A. Adler : A un moment donné, vous avez parlé de la théorie génétique à propos de Mauss; on s'attendrait donc à ce qu'il en développe les grandes phases. Puisqu'il donne une grande place aux Sémites, le premier sacrifice dont il est question est celui de cain et d'Abel. Caín est $1^{\prime}$ agriculteur, Abel le gardien de moutons : celui qui n'a pas d'animal meurt.

J.-P. Colzeyn : Mauss est assez ambigü dans son non-évolutionnisme; quoiqu'il parle d'antêriorité logique, il dresse quand même en ce qui concerne le sacrifice de dieu un schëma avec des producteurs et des produits.

L. de Heusch : Que veut dire consécration ? Est-ce faire participer du "sacrë" ou, au contraire, établir une liaison entre la victime, le sacrifiant et un troisième terme imaginaire?

J. F. Vincent : Il y a aussi l'ambiguité du terme "victime" chez Mauss. Pour lui, il peut s'agir aussi bien de la destruction d'un peu de bière ou de bouillie que de l'immolation d'un animal.

L. de Heusch : Tout 1'Essai est construit sur les sacrifices sanglants, bien qu'il soit dit que le sang n'est pas essentiel.

M. Cartry : Il faudrait étudier aussi les formes d'altération de I'objet. Je pense aux études de G. Dieterlen sur tout ce qui est lié aux processus de fermentation de la bière (les offrandes impliquant une bière fermentée ou non fermentée). 